\title{
Severe Distal Cytomegalovirus Esophagitis With Aerodigestive Symptoms in an Immunocompetent Infant
}

\author{
Nilton Carlos Machado ${ }^{\mathrm{a}, \mathrm{d}}$, Mary A Carvalho ${ }^{\mathrm{a}}$, Erika V P Ortolan ${ }^{\mathrm{b}}$, Maria A M Rodrigues ${ }^{\mathrm{c}}$
}

\begin{abstract}
We report a case of an immunocompetent infant, with no evidence of neurological disorders, which developed clinical manifestation of recurrent crisis of choking, dysphagia, laryngeal stridor and sub costal retractions since the first day of life. Direct laryngoscopy was unremarkable. Upper gastrointestinal series showed a dilated tortuous esophagus with severe peristalsis impairment and reflux episodes till the proximal third of the esophagus. An upper gastrointestinal endoscopy showed a moderately dilated esophagus with erosive lesions in the distal esophagus. Esophageal biopsy specimens revealed CMV inclusion bodies associated to moderate inflammation and immunohistochemistry was positive for CMV early antigen. Prolonged $24 \mathrm{~h}$ esophageal $\mathrm{pH}$ metry was within normal limits. Antiviral therapy with intravenous ganciclovir was introduced and was associated with rapid improvement of the symptoms. Child gradually increased oral intake and weight gain, and there were no side effects related to therapy. Thus, the respiratory symptoms could have been a supra esophageal manifestation of a non-acid reflux disease related to the CMV esophagitis.
\end{abstract}

Keywords: Cytomegalovirus; Esophagitis; Ganciclovir; Immunocompetent infant; Immunohistochemistry

\section{Introduction}

Human cytomegalovirus (CMV) is an opportunistic patho-

Manuscript accepted for publication March 13, 2013

${ }^{a}$ Department of Pediatrics, Division of Pediatric Gastroenterology and Nutrition, Botucatu Medical School, Sao Paulo State University, Botucatu - Sao Paulo, Brazil

${ }^{\mathrm{b}}$ Department of Surgery, Division of Pediatric Surgery, Botucatu Medical School, Sao Paulo State University, Botucatu - Sao Paulo, Brazil

${ }^{\mathrm{c}}$ Department of Pathology, Botucatu Medical School, Sao Paulo State University, Botucatu - Sao Paulo, Brazil

${ }^{\mathrm{d}}$ Corresponding author: Nilton Carlos Machado, Department of Pediatrics, Division of Pediatric Gastroenterology and Nutrition, Botucatu Medical School, UNESP, Sao Paulo State University, Botucatu - Sao Paulo, Brazil. Email: nmachado@fmb.unesp.br

doi: http://dx.doi.org/10.4021/ijcp78w gen that persists in the human host indefinitely after primary infection and may be vertically transmitted from mother to child throughout pregnancy and during or after delivery [1]. CMV determines a wide spectrum of diseases and severe infection is especially reported when disease is congenital, acquired in premature infants or immunocompromised patients [2]. CMV can occur anywhere in the gastrointestinal tract, and immunocompetent infants are usually asymptomatic, although severe gastrointestinal disease has been exceptionally described [3-7]. CMV esophagitis is expressed by luminal syndrome with erosive and ulcerative lesions [8], and there are few reports on severe esophagitis in immunocompetent neonate/infants in the literature review. In two case reports, vomiting, feeding intolerance, failure to gain weight, lymphadenopathy and hepatosplenomegaly were the main symptoms $[9,10]$. One case had severe esophagitis [9] and the other multiple linear erosions and a solitary deep ulcer on the distal esophagus [10]. A third case presented with upper gastrointestinal hemorrhage caused by esophagitis, lack of other manifestations of congenital CMV infection and spontaneous improvement [11]. The aim of this case report is to present a clinical severe aerodigestive manifestation of CMV esophagitis in an apparently immunocompetent infant and the potential role of antiviral treatment.

\section{Case Report}

The child was born from a cesarean section at the gestational age of 37 weeks from healthy non consanguineous parents. Mother was a 35-year-old, G2, P1 and had an uneventful antenatal period, with no drug intake or infection. The first pregnancy was a term healthy girl. The infant boy had a birth weight of $3,010 \mathrm{~g}$ (23.8th percentile), length of $49.5 \mathrm{~cm}$ (42th percentile) and head circumference of $35 \mathrm{~cm}$ (66.4th percentile). The child had no dysmorphic features, and the Apgar score was 6 and 8 at 1 and 5 minutes, respectively. At his first hours of life, after breast feeding he presented clinical evidence of choking, dysphagia and respiratory distress (laryngeal stridor and sub costal retractions) with inadequate ventilation and oxygenation and then, he was transferred to our tertiary care center. On admission, there were no edema, 


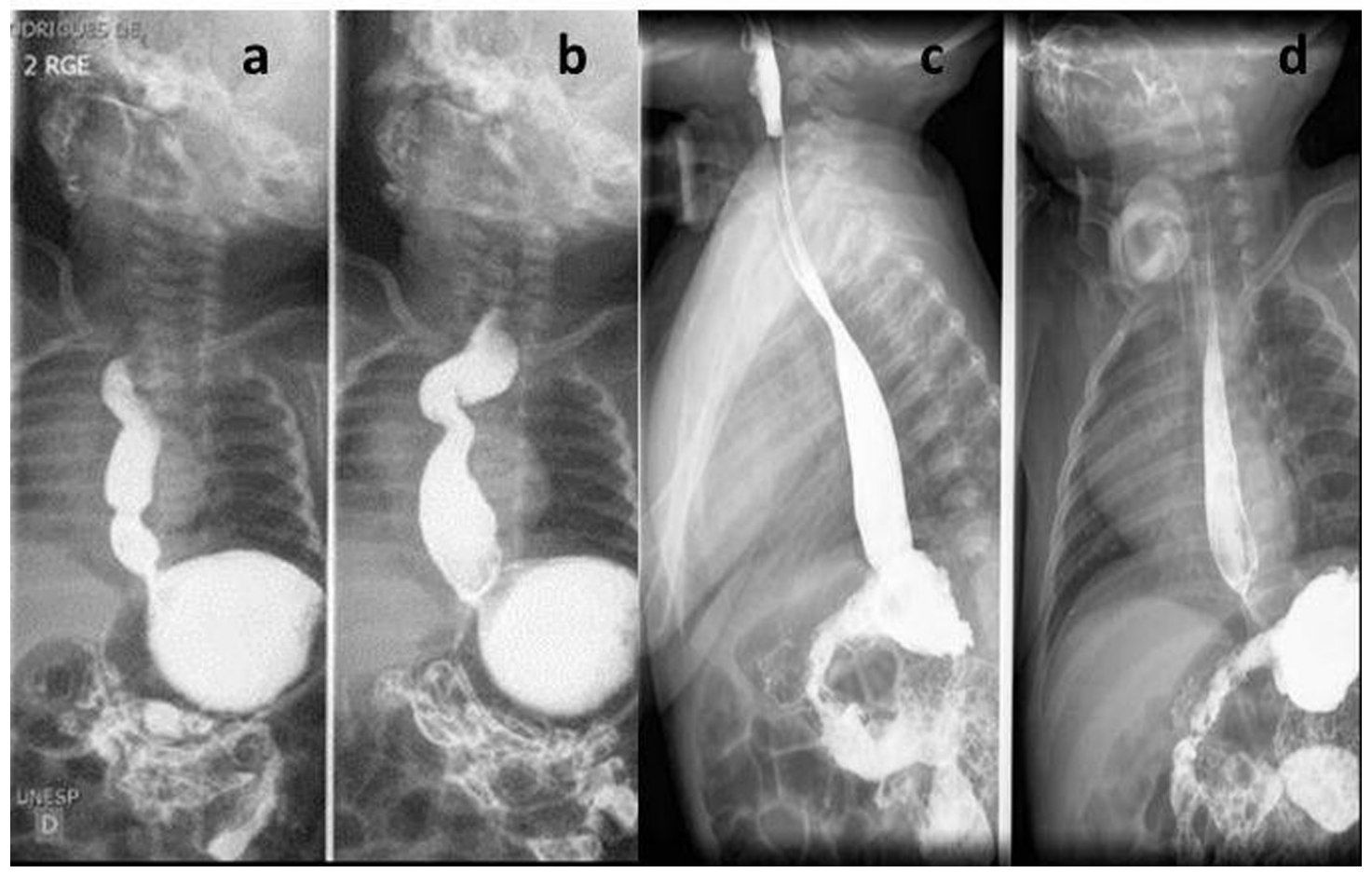

Figure1. Upper gastrointestinal series. Dilated tortuous esophagus with impaired peristalsis $(a, b)$ with improvement after CMV treatment (c, d).

petechiae, rash, jaundice, mouth lesions, lymphadenopathy, hepatosplenomegaly, ascites nor cardiovascular abnormalities. The symptoms and physical examination did not reveal any apparent focus of infection. He developed progressive respiratory distress that required intubation and conventional mechanical ventilation (at the age of $18 \mathrm{~h}$ ).

The laboratory studies demonstrated: hemoglobin level of $177 \mathrm{~g} / \mathrm{L}$, white blood cell count of $19,000 / \mathrm{mm}^{3}$, with a normal differential count and a platelet count of 300,000/ $\mathrm{mm}^{3}$. C-reactive protein level was $0.5 \mathrm{mg} / \mathrm{dL}$ (normal value $<1.0 \mathrm{mg} / \mathrm{dL}$ ). His blood group was $\mathrm{O}$ positive and direct Coombs' test negative. Biochemical analysis including serum electrolytes, glucose, blood urea nitrogen, creatinine, total protein and serum albumin, liver function tests were within the normal range. Cerebrospinal fluid analysis was normal, and culture was negative. The chest X-ray was unremarkable, except for a large thymus.

From the day of life 2 to 10 he required low parameters on mechanical and received gastric feeding with frozen breast milk and a hypoallergenic extensively hydrolyzed formula (Alfare, Nestle). The patient was weaned from continuous mechanical ventilation to nasal continuous positive airway pressure, and from gastric to oral feeding, but laryngeal stridor had again raised requiring re-intubation. The Immunoglobulins IgA, IgG, IgM, and IgE levels were normal. The results of his serological tests for toxoplasmosis, rubella, syphilis, hepatitis B, C, human immunodeficiency virus and Chagas' disease were all negative. Serology revealed a CMV IgG positive and IgM $(1.11 \mathrm{AU} / \mathrm{mL})$ with borderline titer (normal value $<0.9 \mathrm{AU} / \mathrm{mL}$ ). Maternal serology demonstrated CMV IgG positive and IgM negative. Direct laryngoscopy demonstrated mild edema on arytenoids, epiglottis and glottis but no laryngeal, tracheal, vocal folds lesions/ damage or malformations. A cranial, cervical and thorax computed tomographic scan and cervical angiography demonstrated no abnormalities. Electrocardiographic trace and echocardiogram were within normal limits. There were no signs of CMV chorioretinitis on ophthalmological examination, and a newborn hearing screen revealed no deficits.

During the next five weeks, at each attempt of extubation, he developed intense crisis of respiratory distress, choking and dysphagia specially related to attempts of oral feeding, requiring repeated intubations. A plain abdominal radiograph revealed slightly dilated loops of small and large bowel by gas. An upper gastrointestinal series, performed by the oral route, showed a dilated tortuous esophagus with impaired peristalsis and the duodenojejunal flexure well positioned in the left mid abdomen with reflux episodes till the proximal third of esophagus (Fig. 1). Domperidone and ranitidine were introduced with no improvement. At the day of life 51, a tracheostomy was placed for multiple intubation necessity.

At the day of life 52, the pediatric gastroenterology team was called to evaluate the patient. On examination, the child 


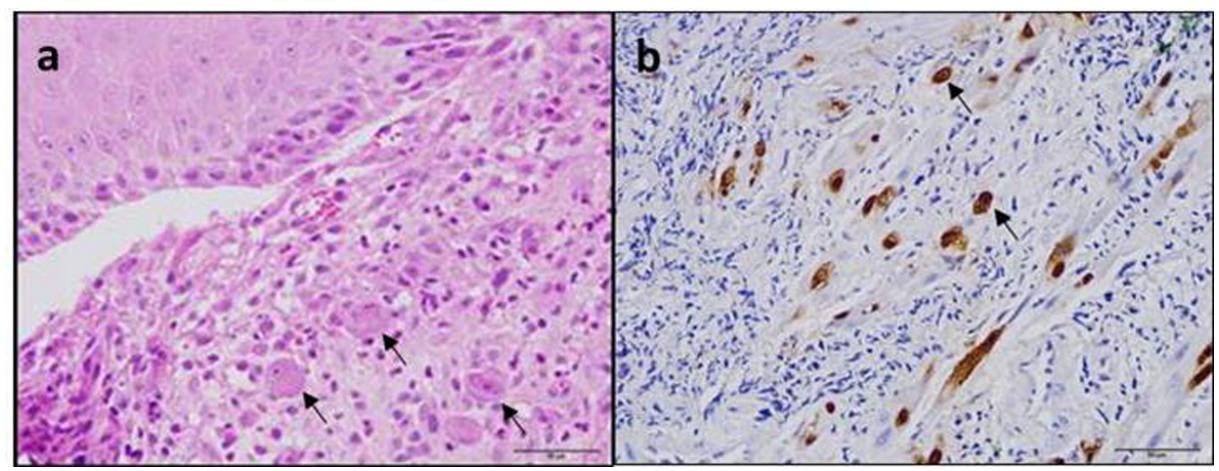

Figure2. Histopathological findings in the distal esophagus. (a) Inflammation and intranuclear inclusions (arrows) in enlarged epithelial cells (haematoxylin-eosin stain, $\times 400$ ) and (b) Cytomegalovirus-infected cells (arrows) with brown coloration in both nuclei and cytoplasm (immunohistochemical staining with anti-CMV).

appeared malnourished and weighed $3.4 \mathrm{~kg}(<3$ rd percentile) with no jaundice, ascites, edema, rash or mouth lesions. The abdomen was soft, with mild distension and no organomegaly. The respiratory, cardiovascular and neurological examination was unremarkable. Prolonged 24h esophageal pH metry was within normal limits. An upper gastrointestinal endoscopy showed a moderately dilated esophagus with erosive lesions in the distal esophagus. Biopsy specimens revealed CMV inclusion bodies associated to moderate inflammation and immunohistochemistry was positive for CMV early antigen (Fig. 2). Antiviral therapy with intravenous ganciclovir was introduced on the 56th day of life at a dose of $10 \mathrm{mg} / \mathrm{kg} / \mathrm{day}$, every 12 hour [12], and maintained for 6 weeks. There were no side effects.

Feeding with breast milk by the oral route was initiated at the 65th day with no signs of chocking or dysphagia. At the end of the treatment, on his 3rd month of life, CMV serology was IgM negative but IgG positive and there was marked improvement of esophageal peristalsis on upper gastrointestinal series (Fig. 2). The upper gastrointestinal endoscopy with biopsy showed no signs of esophagitis. Patient was discharged, and follow-up was maintained in the outpatient clinics for audiological evaluation and programming removal of tracheostomy. At present, having completed his second year of life, his growth, neuropsychological, gross and fine motor skills, age-appropriate visual and nonverbal reasoning and receptive language are normal, with no sensorineural hearing deficits.

\section{Discussion}

We report a case of an immunocompetent infant who developed severe recurrent laryngeal stridor since the first day of life. There are few reports of CMV laryngitis and all describe severe ulcerative laryngitis in immunocompromised patients [13]. In this case, direct laryngoscopy showed only mild laryngeal edema. In the distal esophagus, however, there were
CMV erosive esophagitis, esophageal dilatation and severe peristalsis impairment. Thus, the respiratory symptoms could have been a supra esophageal manifestation of a nonacid reflux disease related to the CMV esophagitis $[14,15]$. CMV treatment was associated with rapid improvement of the symptoms, and the child gradually increased oral intake and weight gain. The prognosis for this immunocompetent infant is good based on the absence of sensorineural hearing deficits and neurologic development [16].

In conclusion, this case is peculiar for the clinical upper aerodigestive presentation, absence of other manifestations of CMV infection and prompt improvement with antiviral therapy. CMV infection is more common than congenital toxoplasmosis, rubella, herpes and other infection groups, although not included in routine prenatal or newborn routine screening [17]. Therefore, pediatricians should be aware of the diversity of locations of tissue-invasive CMV disease on the gastrointestinal tract and include this possibility in the differential diagnosis in immunocompetent infants.

\section{References}

1. Boppana SB, Rivera LB, Fowler KB, Mach M, Britt WJ. Intrauterine transmission of cytomegalovirus to infants of women with preconceptional immunity. N Engl J Med. 2001;344(18):1366-1371.

2. Hamprecht K, Maschmann J, Vochem M, Dietz K, Speer CP, Jahn G. Epidemiology of transmission of cytomegalovirus from mother to preterm infant by breastfeeding. Lancet. 2001;357(9255):513-518.

3. Jonkhoff-Slok TW, Veenhoven RH, de Graeff-Meeder ER, Buller HA. An immunocompetent infant with cow's milk allergy and cytomegalovirus colitis. Eur J Pediatr. 1997;156(7):528-529.

4. Fox LM, Gerber MA, Penix L, Ricci A, Jr., Hyams JS. Intractable diarrhea from cytomegalovirus enterocolitis in an immunocompetent infant. Pediatrics. 
1999;103(1):E10.

5. Quiros-Tejeira RE, Ament ME, Rivera-Penera T, Cortina G, Vargas JH. Cytomegalovirus enterocolitis in an immunocompetent infant host: another cause of treatable intractable diarrhea in infancy. J Pediatr Gastroenterol Nutr. 1999;29(1):86-90.

6. Rongkavilit C, Bedard MP, Ang JY, Asmar BI, Tolia V. Severe cytomegalovirus enterocolitis in an immunocompetent infant. Pediatr Infect Dis J. 2004;23(6):579581.

7. Buonuomo PS, Maurizi P, Valentini P, Mastrangelo S, Lazzareschi I, Ridola V, Riccardi R. Successful treatment with oral valganciclovir in immunocompetent infant with gastrointestinal manifestations of cytomegalovirus infection. J Perinatol. 2006;26(10):648-649.

8. Bobak DA. Gastrointestinal Infections Caused by Cytomegalovirus. Curr Infect Dis Rep. 2003;5(2):101-107.

9. Azimi PH, Willert J, Petru A. Severe esophagitis in a newborn infant. Pediatr Infect Dis J. 1996;15(4):385.

10. Hwang JB, Park MH, Lee BY, Choi WJ, Kim CS, Lee SL, Kang U. Clinical quiz. Cytomegalovirus infection. J Pediatr Gastroenterol Nutr. 2006;42(5):607-608.

11. Weinstein M, Ford-Jones E, Cutz E. Esophagatis and perinatal cytomegalovirus infection. Pediatr Infect Dis J. 2001;20(5):545-546.
12. Schleiss MR. Antiviral therapy of congenital cytomegalovirus infection. Semin Pediatr Infect Dis. 2005;16(1):50-59.

13. Valla F, Leveque N, Escuret V, Galambrun C, Mialou V, Bleyzac N, Bertrand Y. Human cytomegalovirus (HCMV) laryngitis: atypical HCMV disease presentation in haematopoietic stem cell transplantation. J Med Microbiol. 2008;57(Pt 11):1434-1435.

14. Rosen R, Nurko S. The importance of multichannel intraluminal impedance in the evaluation of children with persistent respiratory symptoms. Am J Gastroenterol. 2004;99(12):2452-2458.

15. Johnston N, Wells CW, Samuels TL, Blumin JH. Pepsin in nonacidic refluxate can damage hypopharyngeal epithelial cells. Ann Otol Rhinol Laryngol. 2009;118(9):677-685.

16. Oliver SE, Cloud GA, Sanchez PJ, Demmler GJ, Dankner W, Shelton M, Jacobs RF, et al. Neurodevelopmental outcomes following ganciclovir therapy in symptomatic congenital cytomegalovirus infections involving the central nervous system. J Clin Virol. 2009;46(Suppl 4):S22-26.

17. Johnson J, Anderson B, Pass RF. Prevention of maternal and congenital cytomegalovirus infection. Clin Obstet Gynecol. 2012;55(2):521-530. 